\title{
TRANSPORT PROPERTIES OF DISORDERED POROUS MEDIA FROM THE MICROSTRUCTURE
}

\author{
Progress Report
}

March 1, 1991 - February 28, 1992

\author{
Salvatore Torquato \\ Department of Mechanical and Aerospace Engineering \\ North Carolina State Universitv \\ Raleigh, N. C. 27695-7910
}

November 1991

PREPARED FOR THE U.S. DEPARTMENT OF ENERGY UNDER GRANT NUMBER DE-FG05-86ER13482 


\section{TABLE OF CONTENTS}

I. PROGRESS REPORT FOR THE CURRENT FUNDING PERIOD $\ldots \ldots \ldots \ldots 1$

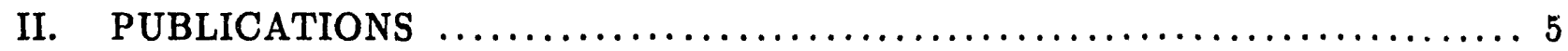

III. INVITED TALKS AND CONFERNECE PRESENTATIONS $\ldots \ldots \ldots \ldots \ldots \ldots 7$

IV. PERSONNEL $\ldots \ldots \ldots \ldots \ldots \ldots \ldots \ldots \ldots \ldots \ldots \ldots \ldots \ldots \ldots \ldots \ldots \ldots \ldots$

V. UNEXPENDED BALANCE FOR THE CURRENT FUNDING PERIOD ..... 9

VI. PLAN OF WORK FOR THE UPCOMING FUNDING PERIOD . .......... 9

VII. BUDGET FOR THE UPCOMING FUNDING PERIOD $\ldots \ldots \ldots \ldots \ldots \ldots \ldots \ldots 11$

\section{DISCLAIMER}

This report was prepared as an account of work sponsored by an agency of the United States Government. Neither the United States Government nor any agency thereof, nor any of their employees, makis any warranty, express or implied, or assumes any legal liability or responsibility for the accuracy, completeness, or usefulness of any information, apparatus, product, or process disclosed, or represents that its use would not infringe privately owned rights. Reference herein to any specific commercial product, process, or service by trade name, trademark, manufacturer, or otherwise does not necessarily constitute or imply its endorsement, recommendation, or favoring by the United States Government or any agency thereof. The views and opinions of authors expressed herein do not necessarily state or reflect those of the United States Government or any agency thereof. 


\section{PROGRESS REPORT FOR THE CURRENT FUNDING PERIOD}

We have had a very productive year. Progress has been made in five general areas: (1) the development of cross-property relations, i.e., relationships between one effective property and a different effective property; (2) the development of a systematic means to represent and calculate statistical correlation functions that arise in rigorous property relations for heterogeneous media, using statistical-mechanical methods; (3) the derivation and calculation of property relations which depend upon this microstructural information; (4) sterically hindered fragmentation in reactive solids using percolation theory and simulations; and (5) the use of Brownian-motion simulation techniques to "exactly" predict the effective properties of disordered media. We embarked on a new area of research, namely, the study of passive advection of tracer particles in random velocity fields. This was one of the problems examined by the P.I. while he was on sabbatical leave at the Courant Institute of Mathematical Sciences from September, 1990 to June, 1991.

During the current funding period, we wrote a total of 16 refereed journal articles. These papers are listed in the "publications" section. The P.I. gave 11 invited lectures on his research work, including 4 at institutions in Europe ( see details below).

\section{A. New Cross-Property Relations}

Researchers have been seeking a rigorous means of connecting the fluid permeability of porous media with some diffusion property of the medium for many years now. Such relations are extremely useful since one then can be determined given the other. We have provided the first rigorous link between the fluid permeability and the electrical or thermal conductivity of porous media [15]. This relation interestingly enough also involves the viscous relaxation times. Since we have shown that the viscous relaxation times are related to the diffusion relaxation times, obtainable from NMR experiments, we can relate the permeability to purely diffusion parameters, i.e., conductivity and diffusion relaxation 
times.

Prior to the work described above, we undertook to study the relationship between the steady-state mean survival time of diffusing Brownian particles in porous media and the aforementioned diffusion relaxation times $[14,16]$. As not:d above, these quantities can be determined from NMR measurements in fluid-saturated porous media.

\section{B. Microstructure Characterization}

We have developed analytical expressions for various types of statistical correlation functions for models of spheres with a polydispersivity in size $[2,5,8]$. We also obtained representations of such correlation functions for anisotropic media composed of distributions of oriented ellipsoids $[2,6]$ which contain as special cases the limits of needles and plates. The nearest-neighbor distribution function $H(r)$ is a fundamental quantity which statistically characterizes a random system of particles (including liquid structure). From $H(r)$ one can compute the mean nearest-neighbor distance and the close-packing density. We continue to study and compute $H(r)$ for nontrivial particulate models $[2,14,16]$.

\section{Effective Property Determinations}

We have computed bounds on a number of different transport properties of realistic models of random media which depend upon the microstructure through various sets of correlation functions. We derived new conductivity bounds for particulate media for the interesting high contrast regimes, e.g., superconducting particles or insulating particles [10]. Bounds on the effective conductivity tensor of sandom arrays of oriented spheroids with arbitrary aspect ratio have been calculated [6]. Conductivity bounds for distributions of equisized spheres have been obtained with heretofore unattained accuracy [1]. We computed bounds on the trapping constant of oriented distributions of parallel spheroids [6]. We also derived new bounds on the trapping constant in terms of a nearest neighbor distribution function and calculated them for the cases of spherical traps [14]. Bounds on 
the fluid permeability tensor of distributions of oriented spheroids have been computed [7]. Finally, bounds on the elastic moduli of suspensions of polydispersed, oriented cylinders have been calculated [3]. Rigorous bounds are generally useful since one of the bounds can provide a good estimate of the effective property, even when the reciprocal bound diverges from it.

Diffusing particles, in actuality, have some finite size: an effect which is typically ignored. We have studied trapping of finite-sized Brownian particles among static traps or sinks $[13,16]$.

We embarked on a new line of research: passive advection by random ve' Specifically, we examined the Lagrangian transport of a passive scalar in a class of incompressible,random stationary velocity fields, termed " random-vortex models". The mean-square displacement and Lagrangian velocity autocorrelation function as functions of time were computed analytically and numerically [11].

\section{Percolation}

A two-dimensional model of a two-phase solid which undergoes a reaction at its surface is employed to study the fragmentation of reactive materials in which the morphological hindering of fragment release is considered [12]. Scaling concepts of cluster percolation theory are utilized to evaluate Monte Carlo data generated from a simulation of the hindered fragmentation process.

\section{E. Brownian-Motion Simulation Technique}

Relatively speaking, there has been a dearth of computer-simulation results which provide "exact" data for the effective properties of random media. The problem has been that traditional techniques (e.g., finite-difference or finite-element methods) often require exorbitant computational costs. Earlier we developed a Brownian-motion simulation technique 
which exactly yields effective properties (governed by a steady-state diffusion equation) in about a tenth of the time required by previous methods. This was done by simulating the "zig-zag" Brownian trajectory of a diffusing particle in the medium. More recently, we modified the simulation procedure by employing first-passage time analysis, eliminating the need to simulate the detailed zig-zag motion in certain domains of the medium and have thus made the methodology at least ten times faster than the original technique. For the irapping problem, this implies that we have reduced the problem from one that was normally solved on a supercomputer to one that can be solved within fifteen minutes on a VAXstation 3100. Thus, using this procedure we will be able to provide important benchmark calculations of effective properties.

During the current funding period, we applied the first-passage time technique to compute the effective conductivity of systems of three-dimensional dispersions of nonoverlapping spheres: much studied models for which there are very few exact results $[4,9]$. We also determined the trapping constant for random arrays of oriented spheroids using the first-passage time simulation methodology [7]. 


\section{PUBLICATIONS}

1. C. A. Miller and S. Torquato, "Effective Conductivity of Hard Sphere Dispersions," Journal of Applied Physics 68, 5486 (1990).

2. S. Torquato, "Random Heterogeneous Media: Microstructure and Improved Bounds on the Effective Properties," Applied Mechanics Reviews, 44, 37 (1991).

3. C. A. Miller and S. Torquato, "Improved Bounds on the Effective Elastic and Transport Properties of Fiber-Reinforced Composites: Effect of Polydispersivity in Fiber Kadius," Journal of Applied Physics, 69, 1948 (1991).

4. I. C. Kim and S. Torquato, "Effective Conductivity of Suspensions of Spheres by Brownian Motion Simulation," Journal of Applied Physics, 69, 2280 (1991).

5. B. Lu and S. Torquato, "General Formalism to Characterize the Microstructure of Polydispersed Random Media," Physical Review A, 43, 2078 (1991).

6. S. Torquato and F. Lado, "Thermal Conductivity, Trapping Constant and the Microstructure of Random Suspensions of Oriented Spheroids," Journai of Chemical Physics, 84, 4453 (1991).

7. C. A. Miller, I. C. Kim and S. Torquato, "Trapping and Flow Among Random Arrays of Oriented Spheroidal Inclusions," Journal of Chemical Physics, 94, 5592 (1991).

8. S. Torquato, "Microstructure and Effective Properties of Random Media ," Lectures in Applied Mathematics, Edited by W. Kohler and B. White, American Mathematical Society, Rhode Island, 27, 323 (1991).

9. I. C. Kim and S. Torquato, "First Passage Time Calculation of the Conductivity of Continuum Models of Multiphase Composites," Physical Review A, 43, 3198 (1991).

10. S. Torquato and J. Rubinstein, "Improved Bounds on the Effective Conductivity of High Contrast Suspensions," Journal of Applied Physics, 69, 7118 (1991). 
11. M. Avellaneda, S. Torquato and I. C. Kim, "Diffusion and Geometric Efffects for Passive Advection by Random Arrays of Vortices "Physics of Fluids A, 3, 1880 (1991).

12. C.A. Miller, A.R. Kerste'n and S. Torquato, "Sterically Hindered Fragmentation in Reactive Solids," Journal of Physics A: Mathematics and General, 24, 3077 (1991).

13. S. Torquato, "Trapping of Finite-Sized Brownian Particles in Porous Media," Journal of Chemical Physics, 85, 2838 (1991).

14. S. Torquato and M. Avellaneda, "Diffusion and Reaction in Heterogeneous Media: Pore Size Distribution, Relaxation Times, and Mean Survival Time," Journal of Chemical Physics, 05, 6477 (1991).

15. M. Avellaneda and S. Torquato, "Rigorous Link Between Fluid Permeability, Electrical Conductivity, and Relaxation Times for Transport in Porous Media," Physics of Fluids A, 3, 2529 (1991).

16. S. Torquato, "Diffusion and Reaction Among Traps: Some Theoretical and Simulation Results," Journal of Statistical Physics, 65, 1173 (1991). 


\section{INVITED TALKS AND CONFERENCE PRESENTATIONS}

1. Random Heterogeneous Media: Structure and Macroscopic Behavior Seminar given at Duke University, Durham, North Carolina, December, 1990.

2. Link Between Flow and Diffusion in Porous Media, Statistical Mechanics Meeting, Rutgers University, New Brunswick, New Jersey, December, 1990.

3. Random Hetrogeneous Media: Microstructure, Diffusion, and Flow, Seminar given at Princeton University, Princeton, New Jersey, February, 1991.

4. Diffusion, Flow and Elastic Behavior of Heterogeneous Materials, Seminar given at the Levich Institute for Physico-Chemical Hydrodynamics, City College of New York, New York, New York March, 1991.

5. Diffusion and Reaction Among Traps: Some Theoretical and Simulation Results, Models for Non-Classical Reaction Rates, National Institutes of Health, Bethesda, Maryland, March, 1991.

6. Transport and Mechanical Properties of Heterogeneous Materials, Exxon Research and Engineering Company, Annandale, New Jersey, May, 1991.

7. Random Heterogeneous Materials: Microstructure and Macroscopic Behavior, Seminar given at the Johns Hopkins University, Baltimore, Maryland, May, 1991.

8. Microstructure and Effective Properties of Random Particulate Media, Euromech C snference on Random Particulate Media, Schumen, Bulgaria, June, 1991.

9. Microstructure and Macroscopic Behavior of Random Heterogeneous Materials, Conference on the Physics of Inhomogeneous Materials, International Center for Theoretical Physics, Trieste, Italy, June 1991.

10. Macroscopic Behavior of Random Inhomogeneous Materials from the Microstructure, Seminar given at the Ecole Polytechnique, Paris, France, June, 1991. 
11. NMR Relaxation in Porous Media, Seminar given at the Institut Francuis du Petrole, Paris, France, June, 1991.

12. Morphology and Macroscopic Behavior of Random Heterogeneous Materials, Seminar given at Princeton University, Princeton, New Jersey, October, 1991. 


\begin{tabular}{l}
\hline \\
DATE \\
FILMED \\
2119192
\end{tabular}


